\title{
VIBRATIONAL CORRECTIONS TO TRANSITION PROPERTIES
}

\author{
T. Pluta \\ Institute of Chemistry, Silesian University, Szkolna 9, 40-006 Katowice, Poland \\ AND A.J. SADLEJ \\ Department of Quantum Chemistry, Institute of Chemistry \\ Nicolaus Copernicus University, Gagarina 7, 87-100 Toruń, Poland \\ (Received August 17, 2000) \\ The vibrational contributions to second-order transition properties are \\ considered in the framework of the Born-Oppenheimer approximation. It is \\ shown that the usual formula for vibrational second-order transition matrix \\ elements is incomplete and needs to be supplemented by a term of purely \\ vibrational origin. This pure vibrational contribution is calculated for vi- \\ brational transition polarizabilities in $\mathrm{LiH}$ and $\mathrm{BeF}$ and found to be quite \\ significant. Its inclusion in theoretically calculated data for the Raman in- \\ tensities appears to be necessary.
}

PACS numbers: $33.20 . \mathrm{Fb}$

\section{Introduction}

Over the past years a great deal of interest [1-3] has been given to vibrational corrections to different molecular properties [4]. Bishop, in his review of the subject [5], stressed that accounting for the influence of molecular vibrations (and rotations) on the calculated molecular properties is much more than just a simple averaging with respect to the given rovibrational $(v, J)$ state. For properties of the second- and higher-orders the usual rovibrational correction $\delta P_{v, J}[6]$, which follows from averaging the so-called electronic contribution, $P^{\text {el }}$, to the total property value $P$ in the given electronic-vibrational-rotational state of the molecule, needs to be supplemented by additional terms, $\delta P^{\text {rv }}$, of rovibrational origin. These terms arise from the fact that in the presence of some external perturbation both the electronic and nuclear motions are affected. Moreover, in several cases $[1-3,7]$ these, usually neglected $\delta P^{r v}$ terms, have been found to make either large or even dominant contribution to the total, i.e., the ones which follow from physical measurements, values of properties. 
It appears that so far most of attention has been focused on vibrational corrections to expectation values of different, mostly electric, properties of molecules. However, a similar mechanism should also add some vibrational and rotational contributions to transition properties. In the present paper we develop a theory of the corresponding corrections to second-order vibrational transition properties. Such a property can be exemplified by the transition dipole polarizability between two vibrational states. This is a quantity which determines the intensity in the vibrational Raman scattering [8]. The magnitude of this effect is first estimated on the basis of spectroscopic parameters. Its magnitude depends on the value of the vibrational transition dipole moment between the two states under consideration and on the difference between vibrational contributions to the dipole moment of these two states. Additional numerical illustration is given by direct calculations of the transition polarizabilities in $\mathrm{LiH}$ and $\mathrm{BeF}$.

\section{Theory}

In this paper we shall focus our attention on a single electronic state derived under the assumption of the validity of the Born-Oppenheimer approximation. Moreover, we shall assume that the overall rotation of the molecule has been separated out and the system is in its rotationless $(J=0)$ state. Transitions between different states of the system will occur between vibrational levels of the same electronic state. For an external perturbation represented in the total electronic-nuclear Hamiltonian $H=H(\lambda)$ by the perturbation term $\lambda V$, whose "strength" is governed by the parameter $\lambda$, the Born-Oppenheimer approximation leads to the notion of the electronic energy $E=E(Q ; \lambda)$ which is an eigenvalue of the electronic Schrödinger equation

$$
H(r ; Q, \lambda) \Phi(r ; Q, \lambda)=E(Q ; \lambda) \Phi(r ; Q, \lambda),
$$

where $r$ denotes the electronic coordinates and $Q$ stands for the set of nuclear geometry parameters. The $\lambda$-dependent Hamiltonian $H(r ; Q, \lambda)$ is

$$
H(r ; Q, \lambda)=H^{0}(r ; Q, 0)+\lambda V .
$$

The eigenvalue of (1) depends on both the nuclear coordinates and on the strength of the perturbation $V$ and defines the $\lambda$-dependent potential in what is called the nuclear Hamiltonian $h=h(Q ; \lambda)$ of the electronic state under consideration

$$
h(Q ; \lambda)=T_{Q}+E(Q ; \lambda),
$$

where $T_{Q}$ is the nuclear kinetic energy operator and $E(Q ; \lambda)$ includes the nuclear repulsion terms. The nuclear eigenvalue problem

$$
h(Q ; \lambda) \chi_{v}(Q ; \lambda)=\epsilon_{v}(\lambda) \chi_{v}(Q ; \lambda),
$$

gives the $\lambda$-dependent solutions which determine the total electronic-vibrational function $\Psi_{v}(r, Q ; \lambda)$ of the electronic state $\Phi(r ; Q, \lambda)$ :

$$
\Psi_{v}(r, Q ; \lambda)=\Phi(r ; Q, \lambda) \chi_{v}(Q ; \lambda) .
$$

If the perturbation $V$ enters the molecular Hamiltonian with some time dependent factor, its presence causes transitions between different electronic-vibrational 
states. The single-quantum vibrational transitions are known to be governed by the (first-order) transition matrix element

$$
P_{v, v^{\prime}}^{(1)}=\lim _{\lambda \rightarrow 0}\left\langle\Psi_{v}(r, Q ; \lambda)\left|\frac{\partial H(\lambda)}{\partial \lambda}\right| \Psi_{v^{\prime}}(r, Q ; \lambda)\right\rangle .
$$

Owing to the assumption of the Born-Oppenheimer approximation one can define the pure electronic contribution to the $\lambda$-dependent first-order transition property (6). In the present case this electronic contribution is simply the expectation value of the operator $V$ in the state $\Phi(r ; Q, \lambda)$ :

$$
P^{(1), \mathrm{el}}(Q, \lambda)=\left\langle\Phi(r ; Q, \lambda)\left|\frac{\partial H(\lambda)}{\partial \lambda}\right| \Phi(r ; Q, \lambda)\right\rangle=\langle\Phi(r ; Q, \lambda)|V| \Phi(r ; Q, \lambda)\rangle .
$$

Thus, upon taking the $\lambda \rightarrow 0$ limit of (6) one obtains the usual vibrational transition element

$$
P_{v, v^{\prime}}^{(1)} \equiv P_{v, v^{\prime}}^{(1), \mathrm{el}}=\left\langle\chi_{v}(Q ; 0)\left|P^{(1), \mathrm{el}}(Q, 0)\right| \chi_{v^{\prime}}(Q ; 0)\right\rangle,
$$

whose determination requires solely the $\lambda$-independent solutions of the electronic and nuclear Schrödinger equations. Because of the Hellmann-Feynman theorem the only vibrational contributions to $P_{v, v^{3}}^{(1), \text { el }}$ are due to $\lambda$-independent vibrational wave functions of the two states involved in the transition. For $V$ being the dipole moment operator this theory gives the usual formula for the vibrational transition dipole matrix element which determines the intensity of the IR bands [9].

In the case of two-quantum transitions caused by the time dependent factor at the perturbation $V$ their intensity is approximately governed [8] by the second-order transition matrix element

$$
P_{v, v^{\prime}}^{(2)}=\lim _{\lambda \rightarrow 0}\left[\frac{\partial}{\partial \lambda}\left\langle\Psi_{v}(r, Q ; \lambda)\left|\frac{\partial H(\lambda)}{\partial \lambda}\right| \Psi_{v^{\prime}}(r, Q ; \lambda)\right\rangle\right] .
$$

By taking the $\lambda \rightarrow 0$ limit of the derivative in (9) one finds that

$$
P_{v, v^{\prime}}^{(2)}=P_{v, v^{\prime}}^{(2), \text { el }}+P_{v, v^{\prime}}^{(2), v i b}
$$

where

$$
P_{v, v^{\prime}}^{(2), \mathrm{el}}=\left\langle\chi_{v}(Q ; 0)\left|P^{(2), \mathrm{el}}(Q ; 0)\right| \chi_{v^{\prime}}(Q ; 0)\right\rangle
$$

and

$$
\begin{gathered}
P_{v, v^{\prime}}^{(2), \text { vib }}=\left\langle\left(\frac{\partial \chi_{v}(Q ; \lambda)}{\partial \lambda}\right)_{\lambda=0}\left|P^{(1), \mathrm{el}}(Q ; 0)\right| \chi_{v^{\prime}}(Q ; 0)\right\rangle \\
\quad+\left\langle\chi_{v}(Q ; 0)\left|P^{(1), \mathrm{el}}(Q ; 0)\right|\left(\frac{\partial \chi_{v^{\prime}}(Q ; \lambda)}{\partial \lambda}\right)_{\lambda=0}\right\rangle .
\end{gathered}
$$

Moreover,

$$
P^{(2), \mathrm{el}}(Q ; 0)=2 \operatorname{Re}\left\langle\left(\frac{\partial \Phi(r ; Q, \lambda)}{\partial \lambda}\right)_{\lambda=0}|V| \Phi(r ; Q, 0)\right\rangle
$$

is the electronic contribution to the second-order property arising from the perturbation $V$.

In Eq. (10) the first term, $P_{v, v^{\prime}}^{(2), \text { el }}$ gives the usual definition (11) of the second-order transition property for transitions between two vibrational states defined by the quantum numbers $v$ and $v^{\prime}$. However, if one takes into account that 
the potential used in the nuclear equation also depends on the external perturbation, there is additional contribution $P^{(2), v i b}$ of purely vibrational origin. This additional term contributing to the total second-order transition property (10) involves the first derivatives of vibrational functions determined at $\lambda=0$. These derivatives are simply the first-order perturbed solutions, $\chi^{(1)}(Q ; 0)$ arising from the perturbation expansion of the $\lambda$-dependent nuclear eigenvalue problem (4). Through the first-order in $\lambda$ one finds

$$
E(Q ; \lambda)=E^{(0)}(Q)+\lambda P^{(1), \mathrm{el}}(Q ; 0)+\ldots
$$

and

$$
\left[h^{(0)}(Q)-\epsilon_{v}^{(0)}\right] \chi_{v}^{(1)}(Q ; 0)=-\left[P^{(1), \mathrm{el}}(Q ; 0)-\epsilon_{v}^{(1)}\right] \chi_{v}^{(0)}(Q)
$$

where the superscript " 0 " indicates the operators and solutions in the absence of the external perturbation, e.g., $\chi_{v}^{(0)}(Q)=\chi_{v}(Q ; 0), \epsilon_{v}^{(0)}=\epsilon_{v}(0)$. Similarly,

$$
\epsilon_{v}^{(1)}=\epsilon_{v}^{(1)}(0)=\left\langle\chi_{v}^{(0)}(Q)\left|P^{(1), \mathrm{el}}(Q ; 0)\right| \chi_{v}^{(0)}(Q)\right\rangle .
$$

In terms of the complete set of solutions $\left\{\chi_{p}^{(0)}\right\}$ of the $\lambda$-unperturbed nuclear problem the first-order vibrational functions can be written as

$$
\chi_{v}^{(1)}(Q)=\chi_{v}^{(1)}(Q ; 0)=-\sum_{p \neq v} \frac{\left\langle\chi_{p}^{(0)}(Q)\left|P^{(1), \mathrm{el}}(Q ; 0)\right| \chi_{v}^{(0)}(Q)\right\rangle}{\epsilon_{p}^{(0)}-\epsilon_{v}^{(0)}} \chi_{p}^{(0)}(Q)
$$

and upon substitution into Eq. (13) give explicit sum-over-states formula for the purely vibrational correction to second-order vibrational transition properties

$$
\begin{aligned}
& P_{v, v^{\prime}}^{(2), \mathrm{vib}}=-\sum_{p \neq v} \frac{\left\langle\chi_{v}^{(0)}(Q)\left|P^{(1), \mathrm{el}}(Q ; 0)\right| \chi_{p}^{(0)}(Q)\right\rangle\left\langle\chi_{p}^{(0)}(Q)\left|P^{(1), \mathrm{el}}(Q ; 0)\right| \chi_{v^{\prime}}^{(0)}(Q)\right\rangle}{\epsilon_{p}^{(0)}-\epsilon_{v}^{(0)}} \\
& -\sum_{p \neq v^{\prime}} \frac{\left\langle\chi_{v}^{(0)}(Q)\left|P^{(1), \mathrm{el}}(Q ; 0)\right| \chi_{p}^{(0)}(Q)\right\rangle\left\langle\chi_{p}^{(0)}(Q)\left|P^{(1), \mathrm{el}}(Q ; 0)\right| \chi_{v^{\prime}}^{(0)}(Q)\right\rangle}{\epsilon_{p}^{(0)}-\epsilon_{v^{\prime}}^{(0)}}
\end{aligned}
$$

Obviously, the usefulness of the sum-over-states expansion of $P_{v, v}^{(2), v i b}$ in its complete form (18) is rather limited. However, in the case of the mechanical and electric harmonic approximations one finds that for each selected mode $v^{\prime}=v \pm 1$ and each of the two sums in Eq. (18) is reduced to a single term. Within these approximations the estimate of (18) will be

$$
\begin{aligned}
& P_{v, v \pm 1}^{(2), \mathrm{vib}} \approx \mp \frac{\left\langle\chi_{v}^{(0)}(Q)\left|P^{(1), \mathrm{el}}(Q ; 0)\right| \chi_{v \pm 1}^{(0)}(Q)\right\rangle}{\omega_{\mathrm{e}}} \\
& \times\left[\left\langle\chi_{v \pm 1}^{(0)}(Q)\left|P^{(1), \mathrm{el}}(Q ; 0)\right| \chi_{v \pm 1}^{(0)}(Q)\right\rangle-\left\langle\chi_{v}^{(0)}(Q)\left|P^{(1), \mathrm{el}}(Q ; 0)\right| \chi_{v}^{(0)}(Q)\right\rangle\right] \\
& \quad=\mp \frac{P_{v, v \pm 1}^{(1), \mathrm{el}}}{\omega_{\mathrm{e}}}\left[P_{v \pm 1, v \pm 1}^{(1), \mathrm{el}}-P_{v, v}^{(1), \mathrm{el}}\right],
\end{aligned}
$$

where $\omega_{\mathrm{e}}$ denotes the harmonic frequency associated with the given mode. This approximate expression for $P_{v, v \pm 1}^{(2), v i b}$ may be helpful in estimating the magnitude of the $P^{(2)}$, vib contribution to vibrational second-order transition properties. The 
evaluation of (19) requires only some lower-order data, i.e., the first-order vibrational transition property and vibrational averages of $P^{(1), \text { el }}$. All these data can be expressed in terms of spectroscopic constants and the first-order derivative of $P^{(1) \text {,el }}$ with respect to the normal coordinate under consideration $[5,6]$. In the case of polyatomic molecules the use of normal coordinates would require the assumption of the small amplitude vibrations. For most of the stable molecules in low rovibrational states this assumption is quite well satisfied.

\section{Applications: transition dipole polarizability}

\subsection{Vibrational transition dipole polarizability}

The formalism presented so far is essentially valid for any second-order vibrational transition property. Its particular application discussed in this section refers to intensities in vibrational Raman spectra [8]. Then, the perturbation operator $V$ can be identified with the negative of some component, say $\widehat{\mu}_{\rho}$, the dipole moment operator

$$
V=-\widehat{\mu}_{\rho} \equiv-\widehat{\mu},
$$

and the strength parameter $\lambda$ acquires the meaning of the $F_{\rho}(=F)$ component of the external electric field. Then, $P^{(1), \text { el }}(Q ; 0)=\mu^{\text {el }}(Q ; 0)$ becomes the electronic part of the dipole moment surface. Similarly, $P^{(2), \text { el }}(Q ; 0)=\alpha^{\text {el }}(Q ; 0)$ is the electronic part of the diagonal $\rho \rho$-component of the dipole polarizability surface. To simplify the notation we shall avoid explicit use of the tensor indices. Analogous identification of symbols holds also for vibrational averages and vibrational transition properties, i.e.,

$$
\begin{aligned}
& P_{v, v}^{(1)} \equiv P_{v, v}^{(1), \mathrm{el}} \Rightarrow \mu_{v, v}, \\
& P_{v, v^{\prime}}^{(1)} \equiv P_{v, v^{\prime}}^{(1), \mathrm{el}} \Rightarrow \mu_{v, v^{\prime}},
\end{aligned}
$$

and

$$
P_{v, v^{\prime}}^{(2)}=P_{v, v^{\prime}}^{(2), \text { el }}+P_{v, v^{\prime}}^{(2), \text { vib }} \Rightarrow \alpha_{v, v^{\prime}}=\alpha_{v, v^{\prime}}^{\mathrm{el}}+\alpha_{v, v^{\prime}}^{\mathrm{vib}}
$$

It is also useful to note that the pure vibrational contribution $\alpha_{v, v}^{\mathrm{vib}}$ to the transition polarizability $\alpha_{v, v^{\prime}}$ can be, according to (13), expressed as the following derivative:

$$
\alpha_{v, v^{\prime}}^{\mathrm{vib}}=\left(\frac{\partial}{\partial F}\left\langle\chi_{v}(Q ; F)\left|-\mu^{\mathrm{el}}(Q ; 0)\right| \chi_{v^{\prime}}(Q ; F)\right\rangle\right)_{F=0},
$$

while $\alpha_{v, v^{\prime}}^{\text {el }}$ is simply a matrix element of $\alpha^{\text {el }}(Q ; 0)$ between the two vibrational states involved in the Raman transition.

The origin of the additional vibrational contribution to the transition dipole polarizability can be also elucidated in the framework of Placzek's theory of the Raman intensities $[8,10]$. In this case one considers the induced electronic dipole moment $\delta \mu^{\mathrm{el}}(Q ; F)$ which arises from the presence of the oscillatory electric field $F$. In the linear approximation one has

$$
\delta \mu^{\mathrm{el}}(Q ; F)=\left(\frac{\mathrm{d} \mu^{\mathrm{el}}(Q ; F)}{\mathrm{d} F}\right)_{F=0} F,
$$


with the total dipole moment derivative approximated by the electronic dipole polarizability, i.e.,

$$
\left(\frac{\mathrm{d} \mu^{\mathrm{el}}(Q ; F)}{\mathrm{d} F}\right)_{F=0} \approx\left(\frac{\partial \mu^{\mathrm{el}}(Q ; F)}{\partial F}\right)_{F=0}=\alpha^{\mathrm{el}}(Q ; 0) .
$$

If one takes into account that the presence of the electromagnetic field influences also the Born-Oppenheimer potential, then the correct expression for the total dipole moment derivative in (25) reads

$$
\begin{aligned}
& \left(\frac{\mathrm{d} \mu^{\mathrm{el}}(Q ; F)}{\mathrm{d} F}\right)_{F=0} \\
& \quad=\left(\frac{\partial \mu^{\mathrm{el}}(Q ; F)}{\partial F}\right)_{F=0}+\left(\frac{\partial \mu^{\mathrm{el}}(Q ; F)}{\partial Q}\right)_{F, Q=0}\left(\frac{\partial Q(F)}{\partial F}\right)_{F=0} .
\end{aligned}
$$

The additional term which accounts for the dependence of normal coordinates on the external field gives rise to the correction $\alpha_{v, v}^{v i b}$ derived in this paper for the static limit of the Raman intensity formulae $[8,10]$.

\subsection{Vibrational contributions to the transition dipole polarizability in $\mathrm{LiH}$ and $\mathrm{BeF}$}

The two molecules, $\mathrm{LiH}$ and $\mathrm{BeF}$, have been selected to exemplify the theory of vibrational contributions to transition dipole polarizabilities. We have calculated potential energy, dipole moment ( $z$-component along the bond), and dipole polarizability ( $z z$-component) curves by using the complete active space self-consistent field (CASSCF) method and the polarized basis sets PolH, PolLi, PolBe, and PolF [11]. The CASSCF calculations [12] have been carried out with active spaces comprising all valence orbitals and the $1 s$ core orbital of the metal atom with 4 and 11 electrons for $\mathrm{LiH}$ and $\mathrm{BeF}$ respectively. These calculations are essentially similar to those carried out earlier by Fowler and one of the present authors [13]. All electric properties needed for the evaluation of the transition dipole polarizability have been obtained by using the finite field perturbation method [14]. The field strength values along the $z$-axis used to obtain finite difference approximations to energy derivatives were $F=0.0, \pm 0.0025$, and \pm 0.0050 a.u.

The present calculations are not aimed at obtaining highly accurate data for any of the studied properties. The main purpose of this numerical illustration is to show the importance of purely vibrational contribution to transition dipole polarizabilities. Since the evaluation of the transition matrix elements requires the energy and property curves to be determined over a rather wide range of interatomic distances, the use of the CASSCF method appears to be quite natural. With the present choice of the active orbital subspaces this method gives a proper dissociation limit for each of the studied molecules.

All parameters needed to estimate the pure vibrational contribution to the transition dipole polarizability according to the approximate formula (19) are summarized in Table I. They are obtained from calculations of the electronic energy and dipole moment curves followed by the solution of the rovibrational problem with the aid of the Numerov-Cooley method $[12,15]$ and calculation of the vibrational dipole moment expectation values and transition dipoles. The electronic 


\section{TABLE I}

Auxiliary parameters for the estimation of the pure vibrational contribution $\alpha_{v, v}^{v i b}$ to vibrational transition polarizabilities $\alpha_{v, v \pm 1}$ in $\mathrm{LiH}$ and BeF. All data in a.u.

\begin{tabular}{c|c|c|c}
\hline & $\mu_{v, v}$ & $\mu_{v+1, v+1}$ & $\mu_{v, v+1}$ \\
\hline $\mathrm{LiH}, \omega_{\mathrm{e}}=1365.3 \mathrm{~cm}^{-1} \approx 0.00622$ a.u. \\
\hline$v=0$ & -2.316 & -2.358 & -0.0892 \\
$v=1$ & -2.358 & -2.400 & -0.1264 \\
\hline \multicolumn{3}{c}{$\mathrm{BeF}, \omega_{\mathrm{e}}=1189.1 \mathrm{~cm}^{-1} \approx 0.00542$ a.u. } \\
\hline$v=0$ & -0.474 & -0.499 & -0.0811 \\
$v=1$ & -0.499 & -0.523 & -0.1156
\end{tabular}

TABLE II

Estimated and directly calculated contributions to transition dipole polarizability ( $z z$-component) in $\mathrm{LiH}$ and BeF. All data in a.u.

\begin{tabular}{cc|c|c|c|c|c}
\hline \hline & & $\alpha_{v, v+1}^{\mathrm{el}}$ & \multicolumn{2}{|c|}{$\alpha_{v, v+1}^{\mathrm{vib}}$} & \multicolumn{2}{|c}{$\alpha_{v, v+1}$} \\
\cline { 4 - 7 } & & & Estimated & Calculated & Estimated & Calculated \\
\hline LiH & $v=0$ & -4.552 & -0.602 & -0.454 & -5.154 & -5.006 \\
& $v=1$ & -7.164 & -0.853 & -0.609 & -8.017 & -7.773 \\
BeF & $v=0$ & -0.333 & -0.372 & -0.247 & -0.705 & -0.580 \\
& $v=1$ & -0.493 & -0.512 & -0.356 & -1.005 & -0.849
\end{tabular}

dipole polarizability curves, $\alpha^{\text {el }}(Q ; 0)$ were used in a similar way to obtain the $\alpha_{v, v^{\prime}}^{\mathrm{el}}$ values presented in Table II.

Table II shows pure vibrational corrections estimated from the data of Table I by using Eq. (19). The results referred to as directly calculated follow from the numerical evaluation of the derivative given by $\mathrm{Eq}$. (24). The matrix elements entering this equation can be easily evaluated from the known dipole moment curve obtained in the absence of the external field and vibrational functions determined in the presence of the external field.

The examples presented in Table II show that the pure vibrational contribution to transition dipole polarizability is by no means negligible. For $\mathrm{LiH}$ it contributes about ten per cent of the total value of $\alpha v, v+1$ for $v$ equal to 0 and 1 . In the case of the $\mathrm{BeF}$ molecule about 40 per cent of the total transition dipole polarizability arises from the purely vibrational term. One has to stress that this term has been so far systematically neglected in evaluations of the Raman intensities. Since the calculation of $\alpha_{v, v}^{\mathrm{vib}}$, for polyatomic molecules may represent quite a task, its simple estimates become quite valuable. The estimation method proposed in this paper is found to give quite reasonable values of $\alpha_{v, v^{\prime}}^{\mathrm{vib}}$. It is of importance that this estimate involves the vibrational transition moment 
and zero-point vibrational corrections $[6,16]$ to the electronic dipole moment in vibrational states linked by the Raman transition, i.e., the quantities which are accessible either experimentally or by computations. The first of these quantities can be either calculated theoretically or determined from infrared band intensities. The calculation of zero-point vibrational corrections to molecular properties is becoming routine $[2,16,17]$. The last parameter, $\omega_{e}$, which enters Eq. (19) is usually known.

\section{Conclusions}

It has been shown that the usual expression for vibrational second-order transition properties needs to be supplemented by additional term of purely vibrational origin. This term arises from the dependence of vibrational functions on the external perturbation and is absent in the case of the vibrational first-order properties. As an example of the theory of vibrational effects on second-order transition properties we have considered vibrational transition dipole polarizabilities. Estimates of the pure vibrational contributions and their directly calculated values show that the so far neglected vibrational effect is by no means negligible and may considerably affect the calculated theoretical data for the Raman intensities.

The present treatment assumes that the transitions under consideration do not involve changes in the rotational state of the molecule. Moreover, it is assumed that zeroth-order perturbation problem is well represented by the harmonic potential. This may to some extent limit the conclusions of the paper making them valid only for relatively low rotational states and restricted to molecules for which anharmonic terms are sufficiently small. The latter restrictions can be easily lifted by using the higher-order perturbation expansions with respect to anharmonic terms of the Born-Oppenheimer potential.

\section{Acknowledgments}

This project was supported by the Committee for Scientific Research under the grant No. 3 T09A 10815.

\section{References}

[1] C.E. Dykstra, S.-Y. Liu, D.J. Malik, Adv. Chem. Phys. 75, 37 (1990) and references therein.

[2] M.J. Cohen, A. Willetts, R.D. Amos, N.C. Handy, J. Chem. Phys. 100, 4467 (1994) and references therein.

[3] M.G. Papadopoulos, A. Willetts, N.C. Handy, A.E. Underlill, Mol. Phys. 88, 1063 (1996).

[4] A.D. Buckingham, Adv. Chem. Phys. 12, 107 (1967).

[5] D.M. Bishop, Rev. Mod. Phys. 62, 343 (1990) and references therein.

[6] A.D. Buckingham, J. Chem. Phys. 36, 3096 (1962); A.D. Buckingham, W. Urland, Chem. Rev. 75, 113 (1975). 
[7] M. Eckert, G. Zundel, J. Phys. Chem. 91, 5170 (1987); D.M. Bishop, J. Pipin, J.N. Silverman, Mol. Phys. 59, 165 (1986); D.P. Shelton, L. Ulivi, J. Chem. Phys. 89, 149 (1988); L. Adamowicz, R.J. Bartlett, J. Chem. Phys. 84, 4988 (1986); L. Adamowicz, J. Chem. Phys. 89, 6305 (1988).

[8] J. Brandmüller, H. Moser, Einführung in die Ramanspektroskopie, Dietrich Steinkopff Verlag, Darmstadt 1963, Ch. 24; D.P. Craig, T. Thirunamachandran, Molecular Quantum Electrodynamics, Academic Press, New York 1984.

[9] K. Nakamoto, Infrared Spectra of Inorganic and Coordination Compounds, Wiley, New York 1963, Ch. 13; S. Califano, Vibrational States, Wiley, New York 1976.

[10] G. Placzek, in: Handbuch der Radiologie, Ed. E. Marx, Akademische Verlag, Leipzig 1934, p. 205.

[11] A.J. Sadlej, Coll. Czech. Chem. Commun. 53, 1995 (1988); A.J. Sadlej, Theor. Chim. Acta 79, 123 (1991); A.J. Sadlej, M. Urban, J. Mol. Struct. (Theochem.) 234, 147 (1991).

[12] K. Andersson, M.R.A. Blomberg, M. Fülscher, V. Kellö, R. Lindh, P.- $\AA$. Malmqvist, J. Noga, J. Olsen, B.O. Roos, A.J. Sadlej, P.E.M. Siegbahn, M. Urban, P.-O. Widmark, Molcas System of Quantum Chemistry Programs, Release 3. Theoretical Chemistry, University of Lund, Lund, Sweden and IBM Sweden, 1994.

[13] P.W. Fowler, A.J. Sadlej, Mol. Phys. 73, 43 (1991).

[14] A.D. McLean, M. Yoshimine, J. Chem. Phys. 46, 3682 (1967); A.D. McLean, M. Yoshimine, J. Chem. Phys. 47, 3256 (1967).

[15] J.W. Cooley, Math. Comp. 15, 363 (1963); C.E. Dykstra, D.J. Malik, J. Chem. Phys. 87, 2806 (1987).

[16] A.J. Russell, M.A. Spackman, Mol. Phys. 90, 251 (1997).

[17] A.J. Russell, M.A. Spackman, Mol. Phys. 88, 1109 (1996). 